\section{Efeitos temporais das estimativas de mortalidade corrigidas de homicídios femininos na Região Nordeste do Brasil}

\section{Temporal effects of corrected female homicide mortality estimates in Northeast Brazil}

\section{Efectos temporales de las estimaciones de mortalidad corregidas de feminicidios en la Región Nordeste de Brasil}

Karina Cardoso Meira 1

Rafael Tavares Jomar 2

Juliano dos Santos 2

Glauber Weder dos Santos Silva ${ }^{3}$

Eder Samuel Oliveira Dantas 4

Ezequiel Benigno Resende 1

Weverton Thiago da Silva Rodrigues 1

Cosme Marcelo Furtado Passos da Silva 5

Taynãna César Simões 6

doi: 10.1590/0102-311X00238319

\section{Resumo}

O objetivo foi analisar os efeitos temporais (idade, período e coorte) na mortalidade por homicídios femininos nos estados da Região Nordeste do Brasil, no período de 1980 a 2017. Estudo ecológico de tendência temporal em que foram utilizados modelos APC com uma abordagem bayesiana e o método determinístico INLA (Integrated Nested Laplace Approximations) na inferência dos parâmetros. As taxas de homicídios femininos, para cada estado da região estudada, foram padronizadas pelo método direito, após correção dos registros de óbitos quanto à qualidade da informação e à subnotificação. Além disso, obtiveram-se dados segundo raça/cor, local de ocorrência e meio pelo qual a agressão foi perpetrada. No período estudado, após a correção dos registros de óbito, a Região Nordeste apresentou taxa média de 5,40 óbitos por homicídios a cada 100 mil mulheres, com aumento significativo em todos os estados nos anos 2000. Em todos os estados houve aumento do risco de óbito $(R R)$ por homicídio na segunda e terceira década de vida e efeito de proteção para as mulheres mais velhas. Com exceção de Sergipe, constatou-se aumento do risco de óbito em quinquênios dos anos 2000. Na Região Nordeste e estados da Paraíba, Pernambuco e Piauí, verificou-se efeito protetor para mulheres de gerações mais antigas. Ainda, a maior proporção de óbitos ocorreu em mulheres negras, no domicílio, sendo perpetrado por arma de fogo. Os achados do presente estudo podem estar correlacionados ao processo de disseminação da violência ocorrido no Brasil, nos anos 2000, assim como a ineficiência do Estado brasileiro em proteger as mulheres vítimas de violência.

Homicídio; Mulheres; Efeito Idade; Efeito Periodo; Efeito de Coortes
Correspondência

K. C. Meira

Av. Senador Salgado Filho 2990, apto. 804, Natal, RN 59075-000, Brasil.

ninameira87@gmail.com

1 Escola de Saúde, Universidade Federal do Rio Grande do Norte, Natal, Brasil.

2 Instituto Nacional de Câncer José Alencar Gomes da Silva, Rio de Janeiro, Brasil.

3 Departamento de Enfermagem, Universidade Federal do Rio Grande do Norte, Natal, Brasil.

4 Hospital Universitário Onofre Lopes, Natal, Brasil.

5 Escola Nacional de Saúde Pública Sergio Arouca, Fundação Oswaldo Cruz, Rio de Janeiro, Brasil.

6 Instituto de Pesquisa René Rachou, Fundação Oswaldo Cruz, Belo Horizonte, Brasil. 


\section{Introdução}

A violência contra as mulheres é perpetrada por parceiros, ex-parceiros, familiares e conhecidos da vítima, manifestando-se como violência psicológica, física, sexual, patrimonial e econômica 1,2,3,4,5. Tais violências muitas vezes se sobrepõem, podendo resultar no feminicídio, a face mais cruel do patriarcado que retira das mulheres o direito à vida 6,7,8,9.

O termo feminicídio foi utilizado pela primeira vez na década de 1970, com objetivo de caracterizar homicídios femininos cometidos por homens cujo principal fator associado é a desigualdade de gênero 10,11. Nos últimos anos, ampliou-se a utilização desse conceito, empregando-o na classificação de todos os homicídios femininos 2,11. Em que pesem os contrapontos desta generalização, ela permitiu maior visibilidade deste agravo à saúde das mulheres, bem como contribuiu para a comparação da tendência temporal do evento entre diferentes países, uma vez que, em muitas regiões, os sistemas de informação sobre mortalidade não discriminam as circunstâncias nas quais o homicídio feminino ocorreu, além de apresentarem problemas de qualidade e cobertura dos registros de óbitos 2,9,11. Isso posto, alguns autores advogam ser possível utilizar a totalidade dos homicídios femininos como uma proxy dos feminicídios em seu conceito original 2,9,11,12,13.

A avaliação da mortalidade por homicídios femininos mostra disparidades importantes na magnitude e tendência temporal entre as regiões do planeta. Entre os anos 2007 e 2012, 54\% das mortes por essa causa se concentraram em 25 países, 14 deles (56\%) localizados na América Central, Caribe e América do Sul 2. Discute-se que tal realidade esteja correlacionada à extrema pobreza, aos conflitos armados por causa de questões agrárias, ao tráfico de drogas, ao contrabando de mercadorias e armas, especialmente em regiões de fronteiras 14,15,16,17,18, que associada à perpetuação da cultura patriarcal e à ineficiência dos países em promover uma rede de atenção e proteção às mulheres em situação de violência, contribui para o maior risco de homicídios femininos nessas regiões.

Estudo de Souza et al. 19 apontou que todas as macrorregiões do Brasil apresentaram tendência ascendente nos coeficientes de homicídios femininos, no período de 1980 a 2014, com exceção da Região Sudeste. A Região Nordeste foi a única a apresentar aumento do risco de morte no quinquênio 2010-2014. É importante destacar que o estudo de Souza et al. pode apresentar taxas subestimadas de mortalidade por homicídios femininos, pois no mesmo período se encontrou tendência ascendente da mortalidade por registros de óbito classificados como evento cuja intenção é indeterminada (ECI), e este estudo não realizou a correção dos registros de óbito para a qualidade da informação e subnotificação dos registros de óbito, que apresenta grande proporção nos estados da Região Nordeste 20.

Destaca-se que, nos anos 2000, foi observado intenso processo de disseminação da violência no Nordeste brasileiro, com ampliação do crime organizado e do tráfico de drogas em seu território, aumentando a violência estrutural e, consequentemente, a violência de gênero 21,22. Esse fenômeno pode estar correlacionado à tendência ascendente de mortalidade e aumento do risco de morte por homicídios em mulheres, inclusive após a promulgação da Lei Maria da Penha 19. Sabe-se que a tendência temporal da morbidade e mortalidade por doenças e agravos à saúde é influenciada por diversos fatores, tais como: (1) estrutura etária da população (efeito da idade); melhoria da qualidade e cobertura dos registros de óbitos, políticas públicas de proteção à mulher em situação de violência, política de segurança pública, políticas de transferência de renda (efeito período); transformações sociais, econômicas e culturais nos hábitos e costumes das populações (efeito de coorte) 23,24,25.

Diante disso, o presente estudo se propôs analisar os efeitos temporais (idade, período e coorte) na mortalidade por homicídios femininos nos estados da Região Nordeste do Brasil no período de 1980 a 2017, após a correção dos registros quanto à qualidade da informação e à subnotificação dos óbitos, a fim de controlar um possível efeito de período promovido por estes artefatos 23,24,25.

\section{Métodos}

Trata-se de um estudo ecológico de avaliação dos efeitos temporais na mortalidade por homicídios femininos a partir da faixa etária de 10 a 14 anos nos estados da Região Nordeste do Brasil, no período de 1980 a 2017. O Nordeste brasileiro é composto por nove estados: Alagoas, Bahia, Ceará, Maranhão, Paraíba, Pernambuco, Piauí, Rio Grande do Norte e Sergipe. 
Os dados de óbitos foram extraídos do Sistema de Informações sobre Mortalidade (SIM) do Departamento de Informática do SUS (DATASUS; http://datasus.saude.gov.br, acessado em Mar/2019), os óbitos referentes à agressão (homicídios) foram obtidos dos códigos E960 a E969 e dos códigos X85 a Y09, respectivamente, na 9a e 10 Classificação Internacional das Doenças (CID).

O presente estudo realizou a correção dos registros quanto à qualidade da informação e à cobertura dos registros de óbitos, pois a evolução temporal da mortalidade por agravos à saúde sofre a influência desses fatores $23,24,25$, pois, embora durante o período de estudo tenham ocorrido melhorias significativas nos registros de óbito no Brasil, especialmente nos anos 2000, ainda há altas proporções de registros de óbitos classificados como ECI e problemas na cobertura dos óbitos nos estados do Nordeste 19,20,26.

Os registros de óbito foram, inicialmente, corrigidos para a qualidade da informação por meio da redistribuição proporcional, segundo ano, faixa etária e estado, com base em quatro etapas 27: (1) calculou-se a proporção dos óbitos por agressão em relação ao total de óbitos por causas externas acidentais e intencionais (lesão autoprovocada, agressão, traumatismos acidentes e intervenção legal); (2) o valor obtido na etapa anterior foi multiplicada pelo total de óbitos classificados como ECI; (3) o resultado da segunda etapa foi adicionado ao total de óbitos originalmente classificados como agressão, no SIM, representando o registro de óbito por agressão corrigido; (4) retificou-se a cobertura dos óbitos por fatores de correção publicados por Queiroz et al. 20, que utilizaram como método de avaliação de cobertura o Synthetic Extinct Generation (SEG), proposto por Bennett \& Horiuchi 27.

Corrigidos os óbitos, calcularam-se taxas brutas e padronizadas pelo método direto de mortalidade por homicídios femininos, tendo como população padrão a população mundial proposta pela Organização Mundial da Saúde (OMS) 29. Os dados populacionais foram obtidos junto ao DATASUS, baseados nos censos populacionais dos anos de 1980, 1991, 2000 e 2010; as projeções intercensitárias para populações para 1o de julho dos anos intercensitários foram estimadas pelo Instituto Brasileiro de Geografia e Estatística (IBGE; https://sidraibge.gov.br/pesquisa/estimapop/tabelas, acessado em 05/Fev/2019).

Também foram extraídos os registros de óbito segundo raça/cor, local de ocorrência e meio pelo qual a agressão foi perpetrada. Utilizaram-se os fatores de correção obtidos na primeira etapa de correção 27 , obtendo-se registros de óbitos corrigidos em relação à qualidade da informação segundo as variáveis descritas anteriormente.

\section{Procedimento de modelagem}

A utilização da inferência bayesiana tem se mostrado indispensável em situações em que a estrutura dos dados apresenta grande complexidade, facilitando a estimação e melhorando as predições, por meio de prioris suavizadas para os efeitos de idade, período e coorte, além de parâmetros para considerar a sobredispersão dos dados, heterogeneidades temporal com e/ou sem estrutura, e testar estatisticamente diferenças entre categorias de variáveis de exposição 30,31,32. Grupos etários e períodos foram agrupados com amplitude de cinco anos, sendo analisadas as faixas etárias de 10-14 a 80 anos e mais, totalizando $I=15$ faixas etárias, períodos de 1980 a 2014 ( $J=7$ períodos), e $K=I+J-1=21$ coortes de nascimento, variando de 1900 a 2000. Optou-se por trabalhar com o intervalo de tempo de 1980 a 2014 para que os períodos fossem comparáveis com o estudo de Souza et al. 19. Destaca-se que, nas figuras, as faixas etárias, os períodos e a coorte foram organizados com os valores medianos, para melhorar a visualização.

Foi ajustado um modelo idade-período-coorte bayesiano univariado (BAPC) para cada estado da Região Nordeste, assim como para a região como um todo 30,31,32. Sendo $y_{i j}$ e $n_{i j}$ o número de óbitos e número de pessoas em risco no grupo etário $i=1, \ldots, 15$, e período $j=1, \ldots, 7$, respectivamente, tem-se o seguinte modelo para cada estado:

$$
\begin{aligned}
y_{i j} & \sim \operatorname{Poisson}\left(n_{i j} \exp \left(\varepsilon_{i j}\right)\right) \\
\varepsilon_{i j} & =\mu_{j}+\alpha_{i}+\beta_{j}+\gamma_{k}+z_{i j}
\end{aligned}
$$

sendo $\mu$ a taxa média global de homicídios em mulheres no período analisado, $\alpha_{i}$ o efeito do grupo etário $i, \beta_{j}$ o efeito do período $j, \gamma_{k}$ o efeito da coorte de nascimento $k$, e $z_{i j} \sim N\left(0, \delta^{-1}\right)$ efeitos aleatórios que incorporam a superdispersão dos dados (variabilidade extra) $30,31,32$. 
Para assegurar identificação do intercepto $\mu$, tem-se a restrição $\sum^{I} \alpha_{i}=\sum^{j} \beta_{j}=\sum^{K} \gamma_{k}=0$. No entanto, por conta do relacionamento linear entre as variáveis temporais, $K=I-i+j$, os efeitos de idade, período e coorte são ainda não identificáveis. Mesmo na abordagem bayesiana, a não identificação dos parâmetros latentes permanece, assim, como nos modelos clássicos, são necessárias restrições adicionais 30,31,32. Uma priori uniforme é atribuída a $\mu$. Os efeitos temporais têm como prioris passeios aleatórios de primeira ordem (RW1) (RW1: $\left.\alpha_{i} \sim N\left(\alpha_{i-1}, k^{-1}\right)\right)$, sendo $k$ os parâmetros de suavização (precisão) aos quais foram atribuídas prioris não informativas $(G(1,0.0005))$.

Para a inferência dos parâmetros, foi utilizado o método determinístico INLA (Integrated Nested Laplace Approximations) 30,31,32. As análises foram feitas no software estatístico R (http://www.r-pro ject.org), com o pacote inla.

\section{Resultados}

No período de 1980 a 2017, foram registrados no SIM 28.205 óbitos por homicídios femininos no Nordeste do Brasil, correspondendo a uma taxa média de 4,13 óbitos por 100 mil mulheres. Após as etapas de correção, houve um aumento de 30,64\% (36.846) nos registros de óbito, aumentando a taxa média para 5,40 óbitos por 100 mil mulheres. Os estados com as maiores taxas médias corrigidas de mortalidade por homicídios femininos a cada 100 mil mulheres foram Pernambuco $(8,29)$ e Alagoas $(7,36)$, enquanto Piauí $(2,93)$ e Maranhão $(3,72)$ apresentaram as menores.

A evolução temporal das taxas de mortalidade por homicídios femininos no período estudado evidenciou aumento percentual acima de 20\% em todos os estados, comparando o primeiro (1980-1984) e o último (2015-2017) períodos. O Nordeste teve aumento de aproximadamente 183,32\%, o Ceará apresentou o maior aumento percentual $(252,1 \%)$ e o Sergipe, a maior redução percentual $(-23,62 \%)$ (Tabela 1).

Tabela 1

Taxas de mortalidade de homicídios femininos não corrigidos e corrigidos para os estados da Região Nordeste, Brasil, no período de 1980 a 2017.

\section{Estado/Taxas}

Alagoas

Taxas de mortalidade sem correção

Taxas de mortalidade corrigidas para

qualidade da informação

Taxas de mortalidade corrigidas para

qualidade da informação e subnotificação

dos óbitos

Bahia

Taxas de mortalidade sem correção

Taxas de mortalidade corrigidas para

qualidade da informação

Taxas de mortalidade corrigidas para

qualidade da informação e subnotificação

dos óbitos

Ceará

Taxas de mortalidade sem correção

Taxas de mortalidade corrigidas para

qualidade da informação

Taxas de mortalidade corrigidas para

qualidade da informação e subnotificação

dos óbitos

$\begin{array}{llllllll}1980-1984 & 1985-1989 & 1990-1994 & 1995-1999 & 2000-2004 & 2005-2009 & 2010-2014 & 2015-2017\end{array}$

\begin{tabular}{|c|c|c|c|c|c|c|c|}
\hline 5,51 & 6,29 & 5,98 & 5,75 & 5,72 & 7,81 & 9,63 & 6,92 \\
\hline 5,60 & 6,18 & 5,66 & 5,62 & 5,50 & 7,41 & 9,45 & 7,01 \\
\hline 5,71 & 6,31 & 5,89 & 5,82 & 6,25 & 8,42 & 10,74 & 7,97 \\
\hline 1,33 & 1,74 & 2,09 & 2,72 & 2,53 & 4,29 & 6,36 & 6,36 \\
\hline 1,35 & 1,75 & 2,11 & 2,70 & 4,21 & 5,98 & 7,86 & 7,53 \\
\hline 1,48 & 1,92 & 2,40 & 3,08 & 4,90 & 7,42 & 9,14 & 8,61 \\
\hline 1,85 & 1,89 & 2,01 & 1,12 & 1,75 & 1,32 & 2,08 & 7,04 \\
\hline 1,96 & 2,42 & 2,52 & 3,18 & 3,66 & 3,85 & 6,06 & 7,91 \\
\hline 2,55 & 3,14 & 3,21 & 4,05 & 4,58 & 4,92 & 7,64 & 8,98 \\
\hline
\end{tabular}

(continua) 


\section{Maranhão}

Taxas de mortalidade sem correção

Taxas de mortalidade corrigidas para qualidade da informação

Taxas de mortalidade corrigidas para qualidade da informação e subnotificação dos óbitos

\section{Paraíba}

Taxas de mortalidade sem correção

Taxas de mortalidade corrigidas para

qualidade da informação

Taxas de mortalidade corrigidas para qualidade da informação e subnotificação dos óbitos

Pernambuco

Taxas de mortalidade sem correção

Taxas de mortalidade corrigidas para

qualidade da informação

Taxas de mortalidade corrigidas para qualidade da informação e subnotificação dos óbitos

Piauí

Taxas de mortalidade sem correção

Taxas de mortalidade corrigidas para qualidade da informação

Taxas de mortalidade corrigidas para qualidade da informação e subnotificação dos óbitos

Rio Grande do Norte

Taxas de mortalidade sem correção

Taxas de mortalidade corrigidas para qualidade da informação

Taxas de mortalidade corrigidas para

qualidade da informação e subnotificação dos óbitos

Sergipe

Taxas de mortalidade sem correção

Taxas de mortalidade corrigidas para

qualidade da informação

Taxas de mortalidade corrigidas para

qualidade da informação e subnotificação dos óbitos

Região Nordeste

Taxas de mortalidade sem correção

Taxas de mortalidade corrigidas para

qualidade da informação

Taxas de mortalidade corrigidas para qualidade da informação e subnotificação dos óbitos

$\begin{array}{llllllll}0,83 & 1,11 & 1,94 & 1,64 & 2,19 & 2,76 & 4,54 & 4,95 \\ 1,15 & 1,31 & 2,13 & 2,74 & 2,59 & 2,99 & 4,93 & 7,71 \\ 2,17 & 2,48 & 3,23 & 4,16 & 2,95 & 3,40 & 5,60 & 8,76\end{array}$

$3,63 \quad 4,34 \quad 2,65$

2,61 
Em todos os estados do Nordeste, verificou-se maior proporção de óbitos em mulheres negras, com prevalência superior a 70\%. Ainda merece destaque o aumento da frequência da via pública como local de ocorrência do óbito ao se comparar o período de 1980-1995 (25,97\%) e 1996-2017 (34\%). No mesmo período, confirmou-se também o aumento da frequência do uso de arma de fogo como meio de perpetração da violência, com destaque para os estados de Alagoas, Paraíba e Pernambuco, em que mais de 60\% dos óbitos no período de 1996 a 2017 foram por tal meio, e nos estados do Maranhão e Piauí, que mantiveram as armas brancas como instrumento mais frequente na perpetração da violência física que resultou em morte.

As maiores taxas de mortalidade no Nordeste ocorreram nas segunda e terceira décadas de vida. Além disso, os maiores coeficientes de mortalidade aconteceram nas coortes mais jovens, variando de 3,98 óbitos (1900-1904) a 6,47 óbitos por 100 mil mulheres (1995-1999). Mantendo-se o mesmo perfil da idade, Pernambuco e Alagoas apresentam as maiores taxas de mortalidade e Piauí e Maranhão, as menores.

No que diz respeito ao efeito da idade estimado pelos modelos BAPC, verificou-se risco estimado de morte (RR) por homicídio feminino variando de 0,20 (10-14 anos) a 2,05 (25-29 anos) no Nordeste (Figura 1). Foram considerados estatisticamente significativos riscos de morte que não contiveram o 1 no intervalo de credibilidade. Com base na avaliação do intervalo de credibilidade, houve efeito protetor para o óbito por homicídios em mulheres no grupo etário de 10 a 14 anos e de 55 a 74 anos. Em contrapartida, houve risco elevado de óbito no grupo etário de 20-24 a 45-49 anos (Figura 1).

\section{Figura 1}

Risco de mortalidade estimado por homicídios femininos e intervalos de 95\% de credibilidade (IC95\%) para o efeito da idade, período e coorte de nascimento. Região Nordeste, Brasil, 1980 a 2014.

1a) Efeito de idade

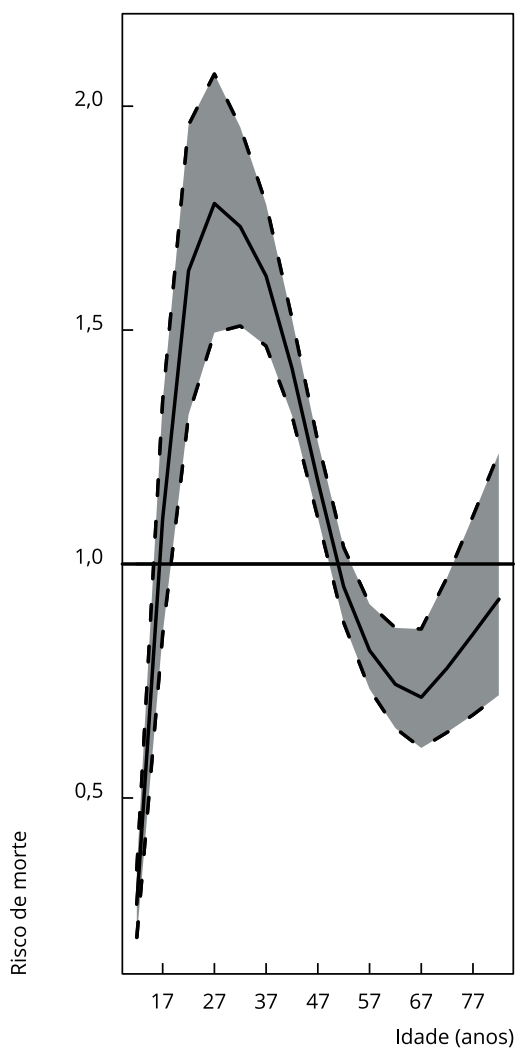

1b) Efeito de período

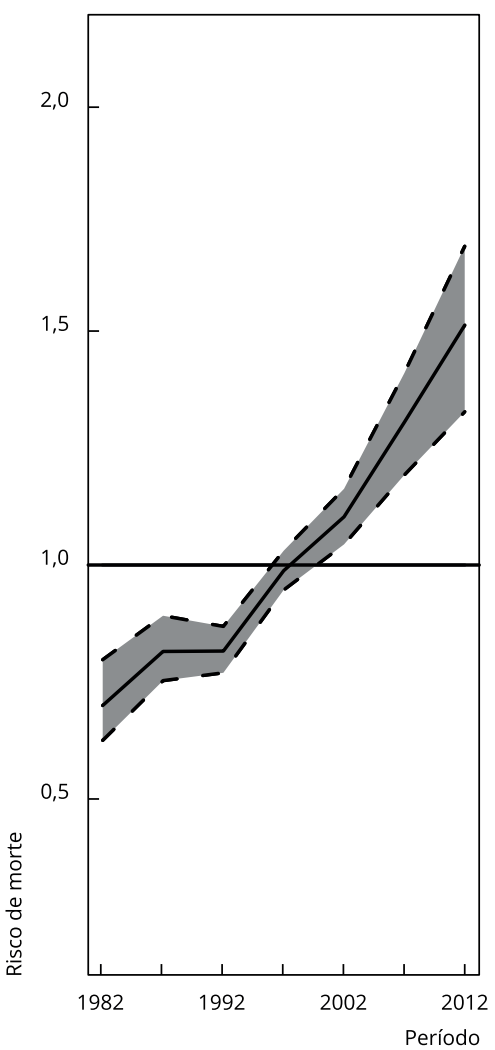

1c) Efeito de coorte

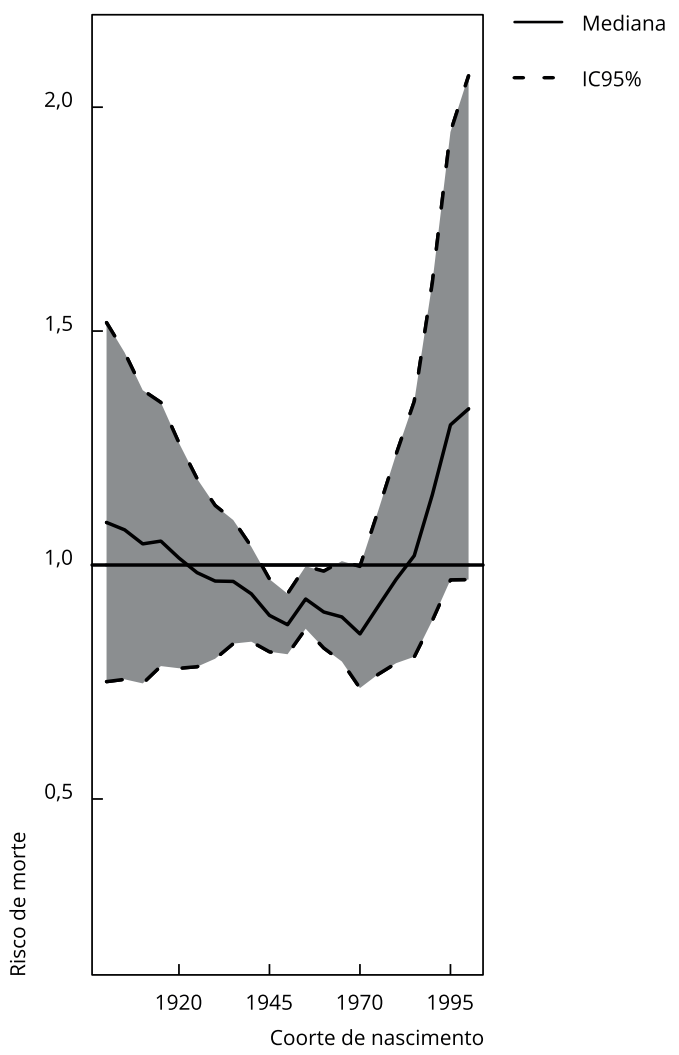


Os estados de Alagoas, Bahia, Ceará, Maranhão e Paraíba mostraram efeito de proteção para a mortalidade por homicídio feminino na faixa etária de 10-14 anos, com aumento do risco estimado nas faixas etárias de 20-24 a 40-44 anos e redução ( $R R<1)$ no grupo etário de 60-64 a 80 e mais anos em Alagoas, 55-64 anos na Bahia e Ceará e 55-74 anos na Paraíba (Figura 2). O efeito da idade no Estado de Pernambuco teve efeito protetor $(R R<1)$ para as idades entre 10 e 14 anos, 55 e 74 anos, e risco de morte $(R R>1)$ de 15 a 44 anos, perfil semelhante ao do Rio Grande do Norte. Em Piauí e Sergipe, também houve efeito de proteção para a faixa etária de 10 a 14 anos, contudo se evidenciou risco elevado de homicídios em mulheres com idades entre 25 e 49, e entre 20 e 39 anos, respectivamente $(\mathrm{RR}>1)$ (Figura 2).

O modelo BAPC mostrou risco estimado de morte segundo período que variou de 0,63 (19801984) a 1,68 (2010-2014) no Nordeste. Houve efeito de proteção ( $R R<1)$ entre 1980 a 1995, com aumento do risco de morte nos quinquênios dos anos 2000 no Nordeste (Figura 1), perfil também encontrado nos estados da Bahia, Ceará e Rio Grande do Norte, embora este último estado tenha apresentado risco no período de 2005 a 2014. Alagoas e Piauí apresentaram redução do risco de óbito na década de 1990 e aumento nos anos 2000, sendo significativo para Alagoas no período de 2005-2009 e para Piauí no período de 2010-2014 (Figura 3). Maranhão e Pernambuco apresentaram

\section{Figura 2}

Risco de mortalidade estimado por homicídios femininos e intervalos de 95\% de credibilidade (IC95\%) para o efeito da idade segundo estados da Região Nordeste, Brasil, 1980 a 2014.

2a) Alagoas

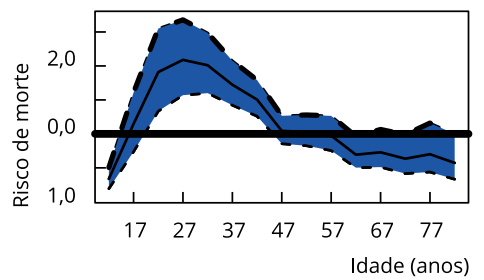

2d) Maranhão

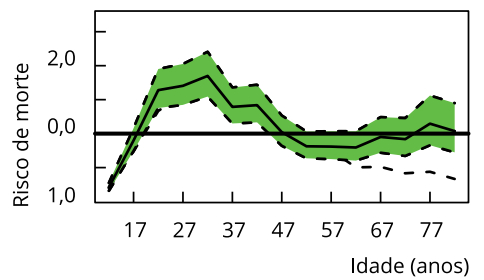

2g) Piauí

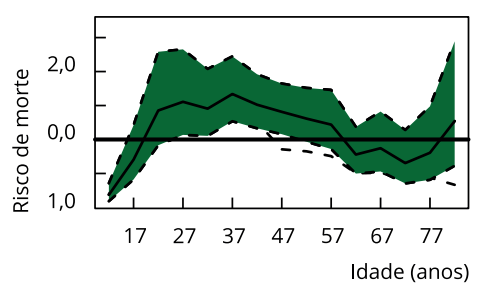

2b) Bahia

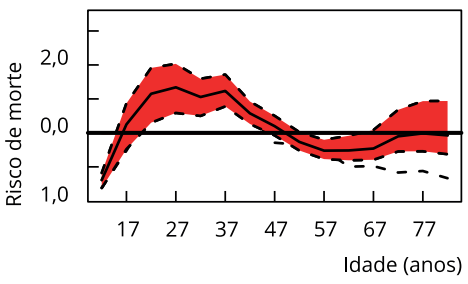

2e) Paraíba

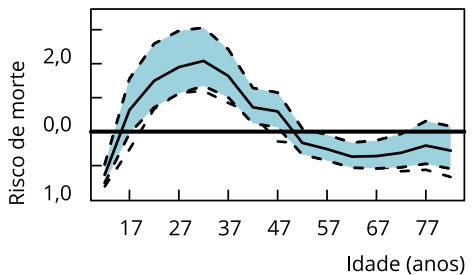

2h) Rio Grande do Norte

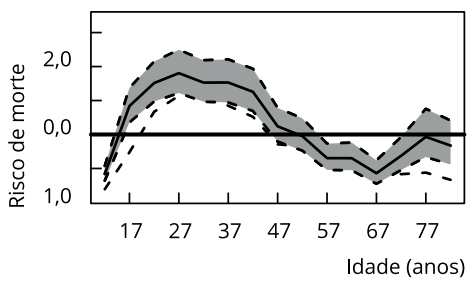

2c) Ceará

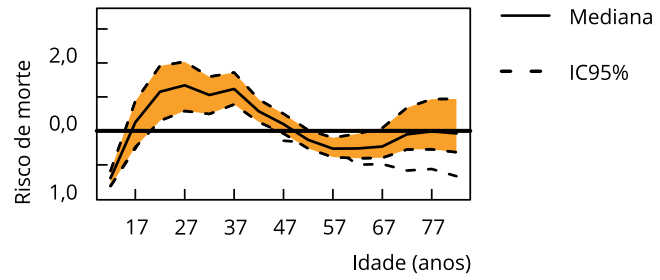

2f) Pernambuco

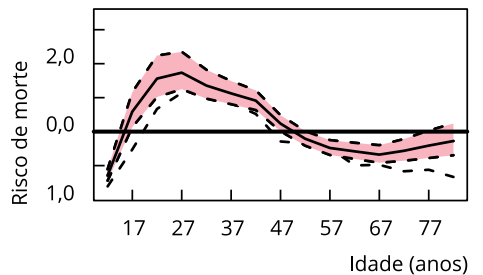

2i) Sergipe

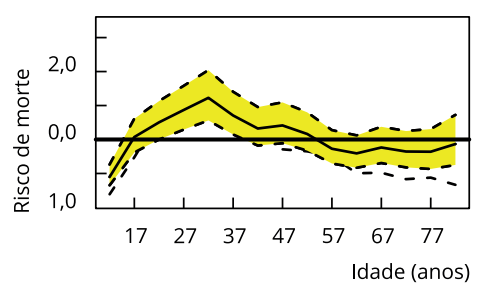


Figura 3

Risco de mortalidade estimado por homicídios femininos e intervalos de 95\% de credibilidade (IC95\%) para o efeito do período segundo estados da Região Nordeste, Brasil, 1980 a 2014.

3a) Alagoas

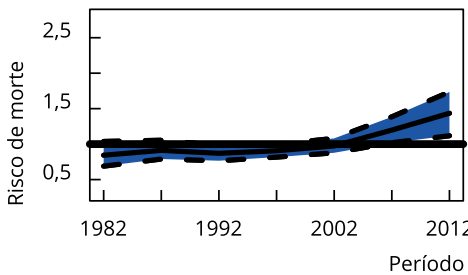

3d) Maranhão

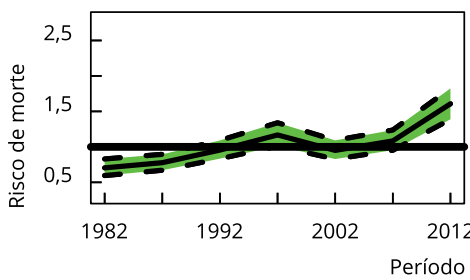

3g) Piauí

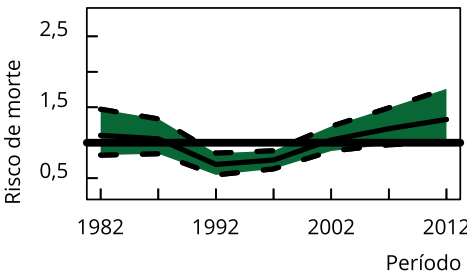

3b) Bahia

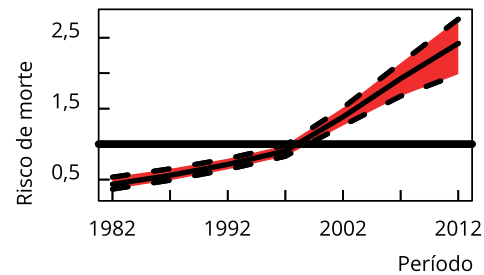

3e) Paraíba

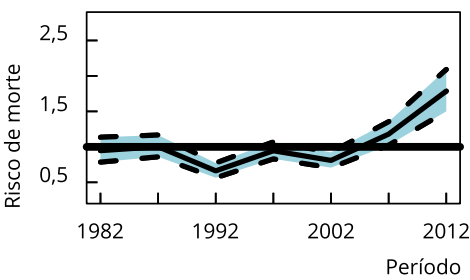

3h) Rio Grande do Norte

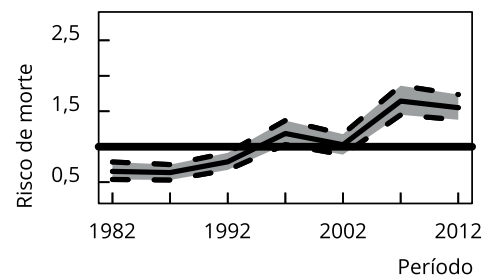

3c) Ceará

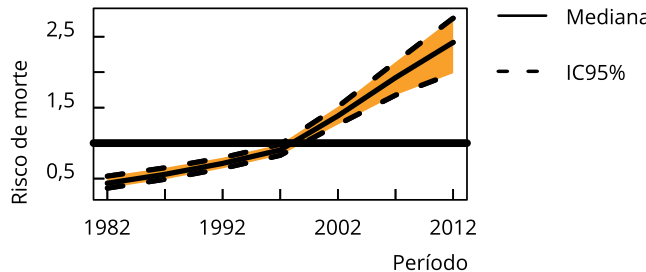

3f) Pernambuco

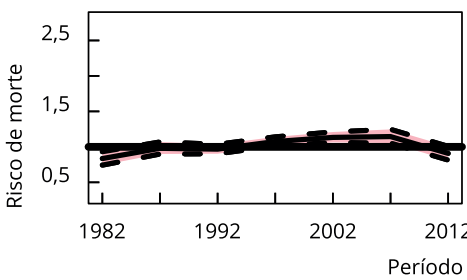

3i) Sergipe

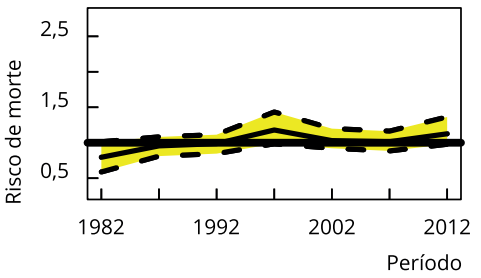

risco de morte (RR > 1) na década de 1980 (1980-1989 e 1980-1984). No Maranhão, houve elevação do risco estimado de óbito nos dois últimos quinquênios da série histórica (2005 a 2014), enquanto em Pernambuco o aumento se deu nos últimos quinquênios da década de 1990 (1995-1999) e dos anos 2000 (2010 a 2014). Divergindo dos demais estados, Paraíba apresentou efeito de proteção para homicídios femininos nos períodos de 1990-1994 e 2000-2004, com elevação do risco de morte no período seguinte (2005-2014) (Figura 3).

O efeito de coorte no Nordeste apresentou risco de morte variando de 0,74 (1970-1974) a 2,05 (2000-2004), com efeito de proteção ( $R R$ < 1) para as mulheres nascidas entre 1934 e 1974 (Figura 1). Nas demais coortes, o risco estimado não foi significativo (valor 1 está contido no intervalo de credibilidade). Do mesmo modo, houve efeito protetor de óbito $(\mathrm{RR}<1)$ para as mulheres nascidas nos estados da Paraíba (1960 a 1969), Pernambuco (1955 a 1974) e Piauí (1935 a 1962). Para as demais gerações, o efeito de coorte não foi significativo, apesar de apresentar uma leve tendência de aumento. Para os demais estados, o modelo BAPC não evidenciou efeito de coorte significativo, enquanto em Alagoas, Bahia e Ceará houve aumento do risco de morte nas gerações da década de 1990 (RR > 1), porém o aumento no risco estimado de morte não foi significativo, de acordo com o intervalo de credibilidade (Figura 4). 


\section{Figura 4}

Risco de mortalidade estimado por homicídios femininos e intervalos de 95\% de credibilidade (IC95\%) para o efeito da coorte segundo estados da

Região Nordeste, Brasil, 1980 a 2014.

4a) Alagoas

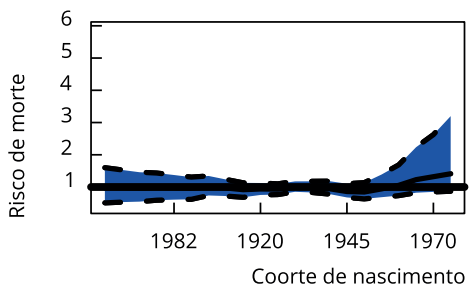

4d) Maranhão

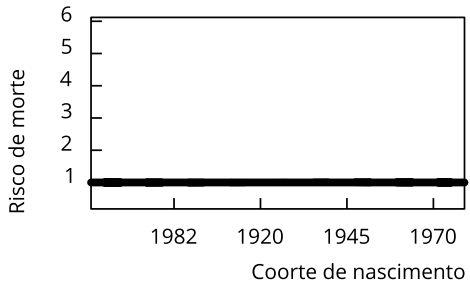

4g) Piauí

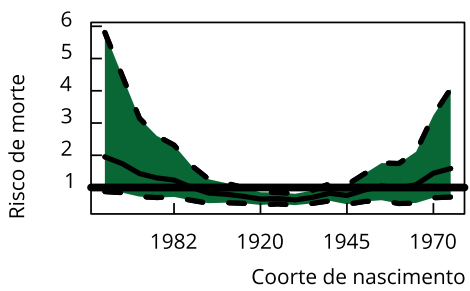

4b) Bahia

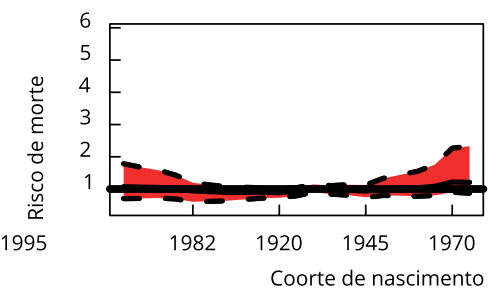

4e) Paraíba

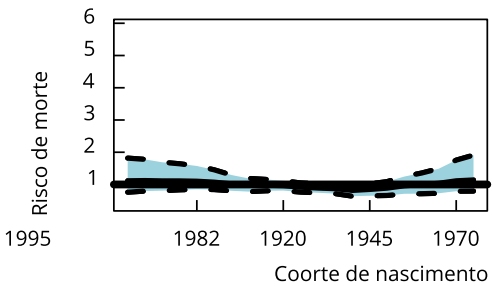

4h) Rio Grande do Norte

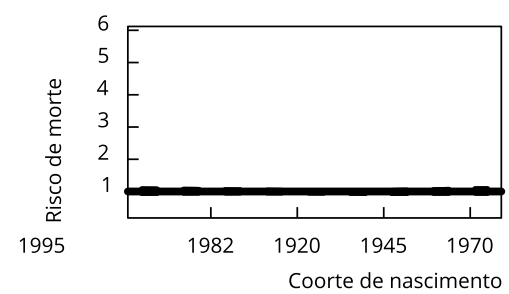

4c) Ceará

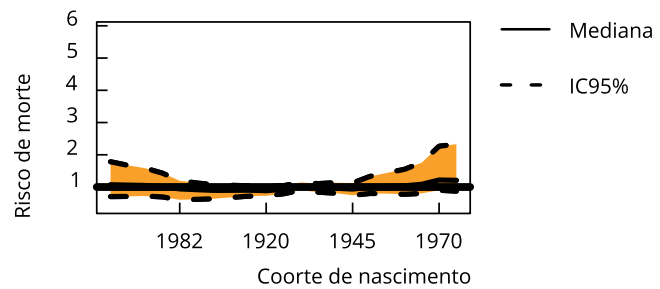

4f) Pernambuco

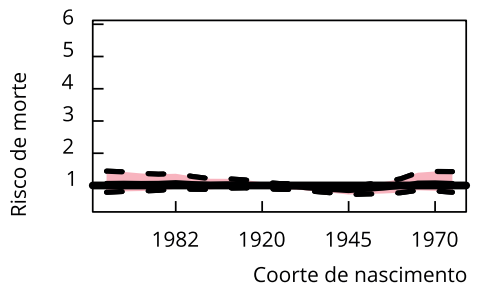

4i) Sergipe

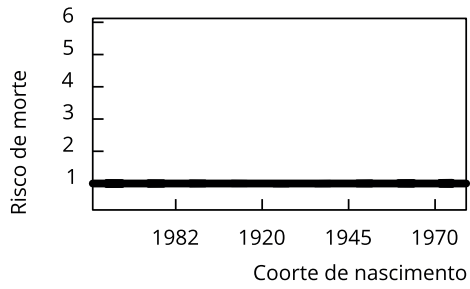

\section{Discussão}

No presente estudo, observou-se aumento nas estimativas de mortalidade por homicídios femininos nos estados do Nordeste do Brasil após a implementação de técnicas indiretas de correção para a qualidade e cobertura dos registros de óbito. A qualidade e a cobertura da certificação dos óbitos são fatores que podem influenciar a tendência temporal da mortalidade por homicídios 19,27,33. Nesse sentido, o estudo de Souza et al. 19 evidenciou que havia uma relação entre as taxas de mortalidade por homicídios femininos com as taxas de mortalidade por registros classificados como ECI em mulheres. Assim, quando havia aumento na magnitude dos coeficientes de um desses grupos de causas, ocorria a redução do segundo, e vice-versa, no período de 1980 a 2014 em todas as macrorregiões brasileiras. Do mesmo modo, evidenciou-se que a redução do risco de morte por homicídios em homens nos anos 2000, observado nos estados de Pernambuco e Rio de Janeiro, foi acompanhado do aumento do risco de óbito classificado como ECI 33 . Resultados esses que reforçam a necessidade da correção dos registros de óbito por causa específica, quando se estuda a tendência temporal da mortalidade em localidades com disparidades importantes na qualidade da informação ao longo do tempo. 
O feminicídio é uma violação aos direitos humanos das mulheres, pois retira delas o direito fundamental à vida e reforça, sobretudo, a cultura machista de dominação masculina em detrimento das mulheres. No Brasil, essas violências que resultam em morte, constituem e permanecem como um problema de extrema importância, visto que mundialmente este país têm o $5^{\circ}$ maior risco de óbitos por homicídios femininos 10, com destaque para as regiões Nordeste e Centro-oeste, que apresentam os maiores coeficientes de mortalidade 11,12,27.

Neste estudo, a Região Nordeste mostrou taxas médias de mortalidade superiores a 3,0 óbitos por 100 mil mulheres em todos os estados, com exceção do Piauí, unindo-se, então, às localidades mais violentas para as mulheres no mundo 2,10. Essa situação torna ainda mais grave e desafiadora quando se discriminam os homicídios femininos por quinquênios, já que houve aumento expressivo nos coeficientes nos anos 2000 para todos os estados, com destaque para os estados de Alagoas e Pernambuco, uma vez que apresentam taxas de mortalidade semelhantes às encontradas em Guatemala e El Salvador: os dois países mais violentos para as mulheres no mundo 2,10.

É importante destacar que as regiões Norte e Nordeste são as macrorregiões de maior vulnerabilidade social do país, além de ser altamente conservadora no que diz respeito aos papéis tradicionais de gênero, em que a cultura da honra é preservada, com a construção da masculinidade baseada na virilidade e heteronormatividade 34 , explicando, em parte, os achados deste estudo. Outro fator que merece ser mencionado é o processo de disseminação e interiorização da violência vivenciado pela Região Nordeste nos anos 2000, promovendo aumento expressivo nas taxas de mortalidade por homicídios em homens 21,22,33 que foi acompanhado pela elevação dos homicídios femininos 9,10,19.

Corroborando tal hipótese, estudos brasileiros demonstraram maiores taxas de mortalidade por homicídios femininos nas macrorregiões brasileiras que estavam em processo de disseminação e interiorização da violência, com correlação positiva entre as taxas de homicídios masculinos e femininos 9,15 .

Meneghel \& Portella 5 afirmam que o feminicídio não se restringe apenas a mortes perpetradas no ambiente doméstico: esse crime ocorre em novas circunstâncias como a agressão sexual, exploração sexual, tráfico de pessoas, mortes por vingança, especialmente em localidades de alta vulnerabilidade social e dominadas pelo crime organizado e tráfico de drogas. Entretanto, os homicídios femininos perpetrados por parceiros íntimos se destacam como o principal contexto de ocorrência, representando 38,6\% dos homicídios femininos em 66 países; ademais, as mulheres têm 6 vezes mais chance de serem assassinadas por parceiros íntimos quando comparadas aos homens 35 .

Frisa-se ainda, que a mortalidade por homicídios em mulheres apresenta grande heterogeneidade em relação à raça/cor, sendo as mulheres negras as que apresentam maior risco de morte por esse grupo de causas. No presente estudo e em outros estudos brasileiros, ressalta-se a grande proporção de óbitos em mulheres afrodescendentes 9,19,27, a descendência da mortalidade por esse grupo de causas de óbitos nas mulheres brancas foi acompanhada da ascendência desses coeficientes em mulheres negras $9,19,27$.

As mulheres negras ocupam a base da pirâmide social no Brasil, e grande proporção delas apresenta os menores níveis de escolaridade, ocupa os postos de trabalho mais precários e residem em regiões de alta vulnerabilidade social.Territórios em que o Estado está mais ausente, regiões muitas vezes dominadas pelo crime organizado, com taxas de mortalidade por homicídios semelhantes às regiões que se encontram em guerra 18,33 .

O risco de morte e as maiores taxas de mortalidade por homicídios femininos nos estados do Nordeste apresentaram-se mais elevados da segunda até a quarta década de vida, assim como em outros estudos 19,36, promovendo grande impacto social e econômico, pois são mulheres em idade reprodutiva, economicamente ativa e que, em muitos casos, têm filhos menores de idade 9,11,13,19,27. Salienta-se que, apesar do aumento das taxas de mortalidade em mulheres idosas nessa região (60-64 e 80 e mais anos), os modelos BAPC revelam efeito de proteção para as mulheres mais velhas, em diferentes faixas etárias em cada estado, particularmente acima dos 55 anos. Tal como os achados de outros estudos que mostraram maior prevalência de violência por parceiro íntimo e homicídios em mulheres jovens 37,38 e maior risco de morte em mulheres nascidas em gerações mais jovens, o presente estudo mostrou redução do risco de morte para as mulheres nascidas até a década de 1970, na Região Nordeste e estados da Paraíba, Pernambuco e Piauí. 
Acredita-se que essa realidade esteja correlacionada ao fato de as mulheres mais jovens terem sofrido o efeito das mudanças socioculturais da revolução sexual e dos costumes ocorridos nas décadas de 1960 e 1970, que passaram a questionar o papel da mulher na sociedade e, portanto, estariam em maior risco de óbito por homicídios porque, ao atrever-se a sair do lugar de subordinação, a mulher desafia a posição do homem, e este, ao se sentir ameaçado, usa da violência, inclusive letal, para voltar a ocupar o papel de dominação que lhe é garantido no sistema patriarcal 3,4,6,7,11,19.

Em razão disso, muito se discute sobre a importância do empoderamento feminino, do acesso das mulheres à educação e à renda, como medida de proteção contra a violência de gênero. Todavia, é importante salientar que a autonomia financeira pode configurar-se como fator de proteção contra a violência doméstica e homicídios em alguns contextos, mas não em outros 38,39. Estudos têm demonstrado que, em regiões de hábitos e costumes mais conservadores, a autonomia financeira das mulheres pode caracterizar-se como uma transgressão, aumentando sua vulnerabilidade à violência doméstica e ao homicídio feminino 38,39. Mulheres residentes na cidade de São Paulo, que relataram autonomia financeira, apresentaram menor chance de violência por parceiro íntimo, inversamente para as residentes na Zona da Mata de Pernambuco onde a autonomia financeira constituiu-se fator de risco $(\mathrm{OR}=1,77$; IC95\%: 1,28-2,45) 38 .

Isso posto, importa discutir a necessidade de uma rede de assistência efetiva por parte do Estado para as mulheres em situação de violência, pois muitas, ao tentarem romper com o ciclo de violência em que vivem, acabam por aumentar a sua vulnerabilidade ao homicídio, tendo em vista que não recebem proteção por parte do Estado. Esperava-se redução dos homicídios femininos nos quinquênios dos anos 2000, especialmente no período de 2005 a 2014, períodos que compreendem respectivamente, a implantação e a vigência da Lei no 11.340/2006 (Lei Maria da Penha) 40, que criou mecanismos para reprimir a violência doméstica e familiar contra as mulheres. No entanto, os resultados deste e de outros estudos têm evidenciado aumento da magnitude das taxas de mortalidade por homicídios femininos nos anos 2000, bem como aumento do risco de morte nos quinquênios dos anos 2000 (RR > 1, como exceção de Sergipe) e elevação da proporção de óbitos ocorridos em via pública 10,13,16,25,28.

Esses resultados podem estar correlacionados ao processo de interiorização e de disseminação da violência vivenciado pelo Nordeste brasileiro, discutido anteriormente, e à ineficiência do Estado em garantir a proteção social das mulheres em situação de violência. Mesmo após a vigência da Lei Maria da Penha, os financiamentos destinados à proteção da mulher em situação de violência são insuficientes para garantir os dispositivos legais garantidos por ela. Gerando quantitativo inadequado de Delegacias de Atendimento à Mulher (DEAM) e Casas-Abrigos. Não têm sido poucos os relatos de mulheres que, mesmo com medidas protetivas, acabam sendo assassinadas por seus ex-parceiros em via pública, pois eles conhecem a rotina das suas ex-companheiras facilitando a perpetração da violência. Estudo realizado por Barufaldi et al. 41 evidenciou que cerca de 15\% dos homicídios femininos registrados no Brasil, no período de 2011 a 2016, ocorreram naquelas que já haviam notificado a violência sofrida, sendo $31 \%$ desses óbitos cometidos em via pública.

Outra questão que merece relevo é o aumento da proporção de óbitos perpetrados por arma de fogo quando se compara o período de 1980-1995 e o de 1996-2017, com aumento importante nos anos 2000, em todos os estados nordestinos, com exceção do Maranhão e do Piauí, em que o instrumento mais frequentemente utilizado foram os perfurantes e penetrantes (facas). Estudos apontam maior proporção de homicídios femininos perpetrados por armas de fogo em localidades com grande circulação de armas 2,19,27. Tais evidências tornam preocupante qualquer medida que facilite a aquisição de arma de fogo no país, já que há maior chance de homicídio para mulheres cujo parceiro íntimo tem acesso à arma de fogo 44. A agressão cometida por arma de fogo apresenta alta letalidade, bastando apenas um disparo para perpetrar a violência letal, enquanto o uso de objetos perfurantes/penetrantes e para estrangulamento/sufocação necessita de grande uso de força por parte do agressor para perpetrar a violência letal, evidenciando um contexto de violência extrema e misoginia 2,11.

Acredita-se que nos próximos anos possa existir manutenção ou aumento dos homicídios femininos no Nordeste brasileiro, pois não há sinalização do Estado em ampliar o financiamento para as estruturas de proteção social às mulheres em situação de violência, visto que está em vigência um ajuste fiscal que contingencia os gastos na área social por 20 anos. Além disso, o Brasil vivencia o fenômeno do backlash, no qual, sob influência do fundamentalismo religioso, declara-se guerra aos direitos das mulheres, defendendo-se a manutenção dos papéis tradicionais de gênero, aumentando a violên- 
cia de gênero e, com ela, os feminicídios, por dar aos homens autorização de usar a força, se necessário, para reocupar o seu lugar na sociedade patriarcal que subjuga as mulheres às suas vontades 43 .

O presente trabalho visa contribuir fornecendo subsídios para avaliação e o planejamento das ações de saúde e segurança pública, para a proteção das mulheres em situação de violência nos estados da Região Nordeste. O processo de correção dos registros de óbito gerou estimativas de mortalidade mais fidedignas. Por sua vez, a utilização de modelos BAPC permitiu a estimação do risco de morte para as faixas etárias, além dos efeitos de período e coorte, e também tem sido o método mais indicado pela literatura para corrigir a principal limitação dos modelos APC (problema de identificação do modelo completo) $30,31,32$.

Como contrapontos deste trabalho, destaca-se a utilização de todos os homicídios femininos como uma proxy para os óbitos decorrentes da violência de gênero, embora estudos prévios tenham empregado a mesma metodologia por conta da ausência de informações sobre as circunstâncias em que os homicídios femininos se deram, e devido aos problemas de cobertura e qualidade da informação, que são corrigidos em parte pelos processos de correção indireto empregados no presente trabalho, a utilização de todos os homicídios femininos compensaria os problemas dos registros de óbitos citados anteriormente e, portanto, não gerariam taxas superestimadas de feminicídio.

\section{Conclusão}

Os resultados do presente estudo apontam para a necessidade de corrigir os registros de óbito por homicídios femininos tendo em vista o aumento significativo dos coeficientes de mortalidade por esse grupo de causas após o processo de correção empregado. Além disso, aponta que os estados do Nordeste brasileiro apresentam magnitude elevada das taxas de mortalidade, sobretudo nos quinquênios dos anos 2000 e em mulheres jovens, sinalizando a gravidade dos homicídios femininos nessa região brasileira, destacando-se como sério problema de saúde pública e desrespeito aos direitos humanos das mulheres.

Sendo assim, são urgentes as medidas para o aumento da proteção social das mulheres em situação de violência e o cumprimento das prerrogativas legais da Lei Maria da Penha por intermédio da ampliação do financiamento, com vistas ao aumento do número de DEAM e Casas-Abrigos, além de capacitar os profissionais do setor saúde, jurídico e social que assistem mulheres em tal situação, a fim de evitar o ciclo da violência doméstica e institucional. Outrossim, são necessárias medidas para que as ordens de restrição sejam cumpridas, assim como celeridade na punição contra os agressores. 


\section{Colaboradores}

K. C. Meira, R. T. Jomar e T. C. Simões contribuíram com a concepção e projeto, análise e interpretação dos dados, redação, revisão crítica relevante do conteúdo intelectual e com a aprovação final da versão a ser submetida. J. Santos contribuiu na concepção e projeto, redação, revisão crítica relevante do conteúdo intelectual e com a aprovação final da versão a ser submetida. G. W. S. Silva, E. B. Resende, W. T. S. Rodrigues e C. M. F. P. Silva contribuíram com a concepção e projeto, análise e interpretação dos dados, redação e revisão crítica relevante do conteúdo intelectual. E. S. O. Dantas contribuiu com a redação, revisão crítica relevante do conteúdo intelectual e com a aprovação final da versão a ser submetida.

\section{Informações adicionais}

ORCID: Karina Cardoso Meira (0000-0002-17225703); Rafael Tavares Jomar (0000-0002-41017138); Juliano dos Santos (0000-0001-9961-3576); Glauber Weder dos Santos Silva (0000-00020570-1944); Eder Samuel Oliveira Dantas (00000002-6595-6105); Ezequiel Benigno Resende (0000-0001-6350-5100); Weverton Thiago da Silva Rodrigues (0000-0001-6411-5390); Cosme Marcelo Furtado Passos da Silva (0000-0001-7789-1671); Taynãna César Simões (0000-0002-5849-343X).

\section{Agradecimentos}

Ao Conselho Nacional de Desenvolvimento Científico e Tecnológico (CNPq) e à Coordenação de Aperfeiçoamento de Pessoal de Nível Superior (CAPES; código de financiamento 001).

\section{Referências}

1. World Health Organization. Multi-country study on womens's health and domestic violence against women. Initial results on prevalence, health outcomes and women's responses. Geneva: World Health Organization; 2011.

2. Alvazzi del Frate A. When the victim is a woman. In: Global Burden of Armed Violence 2011. Geneva: Geneva Declaration Secretariat; 2011. p. 113-44.

3. Safiotti HI. Gênero, patriarcado, violência. São Paulo: Editora Fundação Perseu Abramo; 2004.

4. Carter J. Patriarchy and violence against women and girls. Lancet 2015; 385:1555-6.

5. Meneghel SN, Portella AP. Femicides: concepts, types and scenarios. Ciênc Saúde Colet 2017; 22:3077-86.

6. Taylor R, Jasinski JL. Feminicide and the feminist perspective. Homicide Stud 2011; 15:34162.

7. Karbeyaz K, Yeti ŞY, Güneş A, Şi Mşek Ü. Intimate partner femicide in Eskisehir, Turkey 25 years analysis. J Forensic Leg Med 2018; 60:56-60.

8. Costa MC, Lopes MJM, Soares JSF. Violence against rural women: gender and health actions. Esc Anna Nery Rev Enferm 2015; 19:162-8.

9. Meneghel SN, Rosa BARD, Ceccon RF, Hirakata VN, Danilevicz IM. Femicides: a study in Brazilian state capital cities and large municipalities. Ciênc Saúde Colet 2017; 22:296370.

10. Nowak M. Femicide: a global problem. Small Arms Survey Research Notes 2012; (14):1-4.

11. Meneghel SN, Ceccon RF, Hersler LZ, Margarites AF, Silva SR, Vasconcelos VD. Femicídios: narrativas de crimes de gênero. Interface (Botucatu) 2013; 17:523-33.

12. Garcia LP, Silva GBM. Mortalidade de mulheres por agressão no Brasil: perfil e estimativas corrigidas (2011-2013). Brasília: Instituto de Pesquisa Econômica Aplicada; 2016. (Texto para Discussão, 2179).

13. Garcia LP, Freitas LRS, Hofelmann DA. Avaliação do impacto da Lei Maria da Penha sobre mortalidade de mulheres por agressões no Brasil, 2001-2011. Epidemiol Serv Saúde 2011; 22:383-94.

14. Carcedo A, Sagot M. Femicidio en Costa Rica 1990-1999. Washington DC: Organización Panamericana de la Salud; 2000.

15. Meneghel SN, Hirakata VN. Femicides: female homicide in Brazil. Rev Saúde Pública 2011; 45:564-74.

16. Wright MW. Necropolitics, narcopolitics, and femicide: gendered violence on the MexicoU.S. border. Signs 2011; 36:707-31.

17. Briceño-León R. La comprensión de los homicidios en América Latina: ¿pobreza o institucionalidad? Ciênc Saúde Colet 2012; 17:315970. 
18. Reichenheim ME, Souza ER, Moraes CL, Mello-Jorge MHP, Silva CMFP, Minayo MCS. Violence and injuries in Brazil: the effect, progress made, and challenges ahead. Lancet 2011; 5:69-82.

19. Souza ER, Meira KC, Ribeiro AP, Santos JD, Guimarães RM, Borges LF, et al. Homicides among women in the different Brazilian regions in the last 35 years: an analysis of ageperiod-birth cohort effects. Ciênc Saúde Colet 2017; 22:2949-62.

20. Queiroz BL, Freire FHMA, Gonzaga MR, Lima EEC. Completeness of death-count coverage and adult mortality (45q15) for Brazilian states from 1980 to 2010. Rev Bras Epidemiol 2017; 20 Suppl 1:21-33.

21. Cerqueira D. Atlas da violência 2017. Rio de Janeiro: Instituto de Pesquisa Econômica Aplicada/Fórum Brasileiro de Segurança Pública; 2017.

22. Steeves GM, Petterini FC, Moura GV. The interiorization of Brazilian violence, policing, and economic growth. Economia 2015; 16:359-7.

23. Holford TR. Undestranding the effects of age, period, and cohort on incidence and mortality rates. Annu Rev Public Health 1991; 12:42557.

24. Holford TR. Approaches to fitting age-period cohort models for the Lexis diagram. Stat Med 2006; 26:3018-45.

25. Yang Y, Land KC. Age-period-cohort analysis. New models, methods, and empirical applications. London: Chapman \& Hall/CRC Press; 2013. (Interdisciplinary Statistics Series).

26. Szwarcwald CL, Morais-Neto OL, Frias PG, Souza Júnior PRB, Escalante JJC, et al. Busca ativa de óbitos e nascimentos no Nordeste e na Amazônia Legal: estimação das coberturas do SIM e do SINASC nos municípios brasileiros. Brasília: Ministério da Saúde; 2011.

27. Garcia LP, Freitas LR, Silva GD, Höfelmann DA. Corrected feminicide estimates in Brazil, 2009 to 2011. Rev Panam Salud Pública 2015; 37:251-7.

28. Bennett NG, Horiuchi S. Estimating the completeness of death registration in a closed population. Popul Index 1981; 47:207-21.

29. Mathers CD, Bernard C, Iburg KM, Inoue M, Fat DM, Shibuya K, et al. Global Burden of Disease in 2002: data sources, methods and results. Geneva: World Health Organization; 2003.

30. Riebler A, Held L, Rue H. Estimation and extrapolation of time trends in registry data borrowing strength from related populations. Ann Appl Stat 2012; 6:304-33.

31. Knorr-Held L, Rainer E. Projections of lung cancer mortality in West Germany: a case study in Bayesian prediction. Biostatistics 2001; 2:109-29.

32. Rue H, Martino S, Chopin N. Approximate Bayesian inference for latent Gaussian models by using integrated nested Laplace approximations. J R Stat Soc Series B Stat Methodol 2009; 71:319-92.
33. Borges LF, Souza ER, Ribeiro AP, Silva GWS Silva CMFP, Santos J, et al. Homicídios masculinos em duas regiões brasileiras: análise do efeito da idade, período e coorte. Cad Saúde Pública 2019; 35:e00008719.

34. Souza MGTC, Souza BC, Roazzi A, da Silva ES. Psychocultural mechanisms of the propensity toward criminal homicide: a multidimensional view of the culture of honor. Front Psychol 2017; 8:1872.

35. Stöckl H, Devries K, Rotstein A, Abrahams N, Campbell J, Watts C, et al. The global prevalence of intimate partner homicide: a systematic review. Lancet 2013; 382:859-65.

36. Moreno C, Cendales R. Mortality and years of potential life lost due to homicide in Colombia, 1985-2006. Rev Panam Salud Pública 2011; 30:342-53.

37. Rodrigues NCP, O'Dwyer G, Andrade MKN, Flynn MB, Monteiro DLM, Lino VTS. The increase in domestic violence in Brazil from 2009-2014. Ciênc Saúde Colet 2017; 22:287380.

38. Garcia LP, Duarte EC, Freitas LRS, Silva GDM. Violência doméstica e familiar contra a mulher: estudo de casos e controles com vítimas atendidas em serviços de urgência e emergência. Cad Saúde Pública 2016; 32:e00011415.

39. Koenig MA, Ahmed S, Hossain MB, Khorshed Alam Mozumder AB. Women's status and domestic violence in rural Bangladesh: individual- and community-level effects. Demography 2003; 40:269-88.

40. Brasil. Lei no 11.340, de 7 de agosto de 2006 . Cria mecanismos para coibir a violência doméstica e familiar contra a mulher, nos termos do $\$ 8$ o do art. 226 da Constituição Federal, da Convenção sobre a Eliminação de Todas as Formas de Discriminação contra as Mulheres e da Convenção Interamericana para Prevenir, Punir e Erradicar a Violência contra a Mulher; dispõe sobre a criação dos Juizados de Violência Doméstica e Familiar contra a Mulher; altera o Código de Processo Penal, o Código Penal e a Lei de Execução Penal; e dá outras providências. Diário Oficial da União 2006; 8 ago.

41. Barufaldi LA, Souto RMCV, Correia RSB, Montenegro MMS, Pinto IV, Silva MMAD, et al. Gender violence a comparison of mortality from aggression against women who have and have not previously reported violence. Ciênc Saúde Colet 2017; 22:2929-38.

42. Goodyear A, Rodriguez M, Glik D. The role of firearms in intimate partner violence: policy and research considerations. J Public Health Policy 2020; 41:185-95.

43. Faludi S. Backlash: o contra-ataque na guerra não declarada contra as mulheres. Rio de Janeiro: Rocco; 2001. 


\section{Abstract}

This study aimed to analyze the temporal effects (age, period, and cohort) on female homicide mortality in the states of Northeast Brazil from 1980 to 2017. This ecological time trend study used APC with a Bayesian approach and the deterministic method Integrated Nested Laplace Approximations (INLA) in the parameters' inference. The female homicide rates for each state of the Northeast were standardized by the direct method after correction of the death records for quality of information and underreporting. Data were also obtained on race/color, place of death, and means of perpetration. During the period analyzed, after correcting the death records, the Northeast region showed a mean rate of 5.40 female homicide deaths per 100,000 women, with a significant increase in all the states in the 2000s. In all the states, there was an increase in relative risk $(R R)$ of homicide death in the second and third decades of life and a protective effect in older women. Except for the state of Sergipe, there was an increase in the risk of death in all five-year periods in the 2000s. The Northeast region as a whole and the states of Paraíba, Pernambuco, and Piaui showed a protective effect for women from older generations. There were also higher proportions of deaths in black women, homicides committed at home, and those perpetrated with firearms. The current study's findings may correlate with the spread of violence in Brazil in the 2000s and the Brazilian State's failure to protect women from violence.

\section{Homicide; Women; Age Effect; Period Effect;} Cohort Effect

\section{Resumen}

Analizar los efectos temporales (edad, período y cohorte) en la mortalidad por feminicidios en los estados de la región Nordeste de Brasil durante el período de 1980 a 2017. Estudio ecológico de tendencia temporal en el que se utilizaron modelos $A P C$ con un abordaje bayesiano y el método determinístico INLA (Integrated Nested Laplace Approximations) para la inferencia de los parámetros. Las tasas de feminicidios, para cada estado de la región estudiada, fueron estandarizadas por el método directo, tras la corrección de los registros de fallecimientos, en cuanto a la calidad de la información y a la subnotificación. Asimismo, se obtuvieron datos según raza/color, lugar de ocurrencia y medio por el que se perpetró la agresión. Durante el período estudiado, tras la corrección de los registros por fallecimiento, la región Nordeste presentó una tasa media de 5,40 óbitos por feminicidio en cada 100.000 mujeres, con un aumento significativo en todos los estados a partir del año 2000. En todos los estados hubo un aumento del riesgo de óbito (RR) por homicidio en la segunda y tercera década de vida y efecto de protección para las mujeres mayores. Con excepción del estado de Sergipe, se observó un aumento del riesgo de fallecimientos en quinquenios de los años 2000. En la región Nordeste y estados de Paraíba, Pernambuco y Piauí se verificó un efecto protector para las mujeres de generaciones mayores. Asimismo, la mayor proporción de fallecimiento se produjo en mujeres negras, en el domicilio, siendo perpetrado por arma de fuego. Los resultados del presente estudio pueden estar correlacionados con el proceso de diseminación de la violencia, ocurrido en Brasil a partir del año 2000, así como la ineficiencia del Estado brasileño para proteger a las mujeres víctimas de violencia.

Homicidio; Mujeres; Efecto Edad; Efecto Periodo; Efecto de Cohortes
Recebido em 12/Dez/2019

Versão final reapresentada em 06/Mai/2020

Aprovado em 29/Jun/2020 